\section{HIV-1 subtype and second-receptor use}

SIR - The identification of the chemokine receptors fusin (now renamed CXCR4) and CCR5 as the main coreceptors for HIV-1 entry into $\mathrm{CD}^{+}{ }^{+}$cells of the immune system will assist our understanding of HIV-1 transmission and pathogenesis $^{1-6}$. Here we report data showing that the phenotype of the virus, rather than its genetic subtype, determines receptor usage.

The phenotype of a virus is closely associated with whether it preferentially uses CXCR4 or CCR5: NSI viruses, which are macrophage-tropic and do not is also a more divergent set of viruses known as the outlier $(\mathrm{O})$ group. It is possible that different genetic subtypes might preferentially use different co-receptors, hence contributing to the uneven worldwide distribution of viral subtypes. The only recent report on this topic indicates that virus strains from subtypes $\mathrm{A}, \mathrm{C}$ and $E$ can use CCR5, although only a few viruses were tested ${ }^{5}$.

We therefore examined 18 viral isolates from the HIV-1 M group (at least three for each of subtypes A, B, C, D and E), and two from the $\mathrm{O}$ group, for second-

SECOND-RECEPTOR USAGE BY DIFFERENT GENETIC SUBTYPES OF HIV-1

p24 production in HOS.CD4 cells*

p24 production in PBMC

Virus Subtype Phenotype CCR1-4 $4^{\dagger}$ CCR5 CXCR4 PB wt/wt wt/ $\Delta 32 \quad \Delta 32 / \Delta 32^{\S}$

93KE101

93 IN103

92RW009

$92 \mathrm{HA} 593$

$92 \mathrm{HA} 594$

$92 \mathrm{HA} 596$

JRCSF

JRFL

$\mathrm{NL} 43$

92ZW101

$92 Z \mathrm{~W} 102$

92ZW106

94KE102

$94 K E 103$

92 UG046

93TH304

93TH305

93TH307

CA9

MVP5180
A NSI

A NSI

A

B

B

B

B

B

C

C

C

D

D

D

E

$E$

E

0
NSI

SI

SI

SI

NSI

NSI

SI

NSI

$\mathrm{NSI}$

SI

NSI

NSI

$\mathrm{SI}$

SI

NSI

NSI

NSI
SI

$\begin{array}{ll}- & + \\ - & + \\ - & + \\ - & + \\ - & + \\ - & + \\ - & + \\ - & + \\ - & - \\ - & + \\ - & + \\ - & - \\ - & + \\ - & + \\ - & + \\ - & + \\ - & + \\ - & + \\ - & + \\ - & +\end{array}$

* Plus symbol, p24 concentration $>60 \mathrm{pg} \mathrm{ml}^{-1}$; minus symbol, $\leqslant 10 \mathrm{pg} \mathrm{ml}^{-1}$

${ }^{+}$Identical results were obtained with CCR1, 2, 3 and 4.

${ }^{\ddagger}$ Negative control cells containing vector pBABE-puro only ${ }^{2}$.

$\S$ Identical results were obtained with PBMC from two cases.

induce syncytia, use CCR5, whereas SI strains, which induce syncytia or are adapted to T-cell lines, prefer CXCR4. There is one example of a broadly tropic HIV-1 strain able to use both CCR5 and CXCR4 for entry ${ }^{4}$. In addition, CCR1, CCR2b and CCR3 can function to a limited extent as HIV-1 co-receptors for some viral strains ${ }^{3-5}$. With a single exception $^{5}$, these studies used HIV-1 strains or env genes derived solely from genetic subtype B, the subtype of HIV-1 that predominates in North America and Europe but not globally. Nine HIV-1 genetic subtypes (A to I) have now been designated within the major (M) group, and there

\section{Scientific Correspondence}

Scientific Correspondence is intended to provide a forum in which readers may raise points of a scientific character. Priority will be given to letters of fewer than 500 words and ten references. receptor usage. These $20 \mathrm{HIV}-1$ isolates, originating from 8 different countries, had previously been genetically subtyped and their NSI or SI phenotypes determined in MT-2 cells. To assess second-receptor use, we added 500 TCID $_{50}$ (half-maximal tissue-culture infectious dose) of each isolate to HOS cells engineered to express human CD4 and CCR1, CCR2b, CCR3, CCR4, CCR5 or CXCR4 (ref. 2). We assessed virus replication using supernatant p24 antigen concentration on day 6 after infection.

We found that each NSI isolate could utilize CCR5 for entry, irrespective of its genetic subtype (see table). However, SI isolates could use both CCR5 and CXCR4, except for one subtype B TCLA virus (NL43) and one subtype C SI primary isolate (92ZW106), which could only utilize CXCR4. The finding that dualreceptor usage is more common among SI strains suggests that adaptation of HIV-1 to use CXCR4 during phenotypic evolu- tion may not be at the expense of losing the ability to use CCR5. Alternatively, it is possible that most SI isolates contain a mixed population of SI and NSI quasispecies, and thus seem to be able to use both CCR5 and CXCR4.

To confirm our observations on HOS cells, we tested the ability of isolates from different subtypes to replicate in peripheral blood mononucleocytes (PBMC) from individuals homozygous or heterozygous for a recently identified defective allele of CCR5 that contains a 32-nucleotide deletion $(\Delta 32)$ in the second external loop and encodes a non-functional protein for HIV1 entry $^{7,8}$. We reasoned that if isolates from non-B subtypes could use cofactors other than CCR5 for entry into PBMC, they would replicate in the cells of $\triangle 32$ homozygotes. We therefore tested the same panel of viruses for replication in stimulated PBMC from two homozygous $(\Delta 32 / \Delta 32)$, one heterozygous $(w t / \Delta 32)$ and one wild-type (wt/wt) individuals (see table). No NSI isolate, irrespective of the genetic subtype, could replicate in PBMC from $\Delta 32$ homozygotes, but all replicated efficiently in wild-type and heterozygote PBMC. In contrast, every SI isolate could replicate in all PBMC regardless of CCR5 genotype, presumably by using CXCR4. Thus, there is an absolute requirement for the presence of a functional CCR5 allele for replication of NSI strains of all the genetic subtypes tested.

We conclude that CCR5 and CXCR4 are the main co-receptors for HIV-1 subtypes A-E and group O. Viral phenotype is dominant over genotype in terms of co-receptor usage. Thus, the uneven worldwide distribution of HIV-1 subtypes is more likely to be the result of stochastic dissemination. However, it is conceivable that sequence polymorphisms in CCR5 and CXCR4 in different human populations could affect the efficiency of HIV-1 entry in a subtype-dependent manner. Finally, the common usage of CCR5 and CXCR4 by multiple genetic subtypes suggests that drugs targeting these receptors might not be unduly compromised by HIV-1 genetic diversity.

\section{Linqi Zhang}

Yaoxing Huang

Tian He

\section{Yunzhen Cao}

David D. Ho*

Aaron Diamond AIDS Research Center, The Rockefeller University,

New York,

New York 10016, USA

e-mail: dho@adarc.org

$$
\begin{aligned}
& \text { *To whom correspondence should be addressed. } \\
& \text { 1. Feng, Y., Broder, C. C., Kennedy, P. E. \& Berger, E. A. } \\
& \text { Science 272, 872-877 (1996). } \\
& \text { 2. Deng, H. et al. Nature } \mathbf{3 8 1}, 661-666 \text { (1996). } \\
& \text { 3. Dragic, T. et al. Nature 381, 667-673 (1996). } \\
& \text { 4. Doranz, B. J. et al. Cell 85, 1149-1158 (1996). } \\
& \text { 5. Choe, H. et al. Cell 85, 1135-1148 (1996). } \\
& \text { 6. Alkhatib G. et al. Science 272, 1955-1958 (1996). } \\
& \text { 7. Liu, R. et al. Cell 86, 367-377 (1996). } \\
& \text { 8. Samson, M. et al. Nature } \mathbf{3 8 2}, 722-725 \text { (1996). }
\end{aligned}
$$

